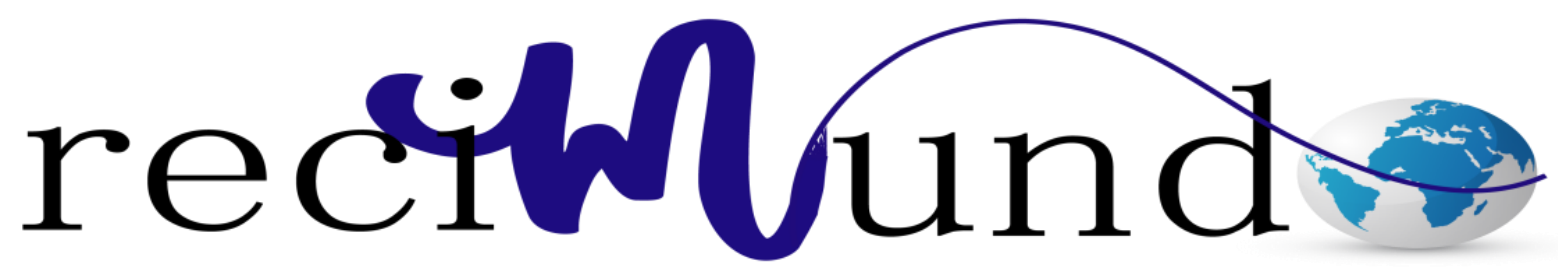

Revista Científica Mundo de la Investigación y el Conocimiento

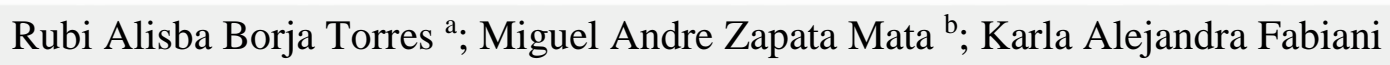
Portilla $^{\mathrm{c}}$; Genesis Ariana Borja Torres ${ }^{\mathrm{d}}$

Eficacia de la escala Alvarado y Ripasa en el diagnóstico oportuno y el control de complicaciones de la apendicitis aguda

Efficacy of the Alvarado and Ripasa scale in the timely diagnosis and the control of complications of acute appendicitis

Revista Científica Mundo de la Investigación y el Conocimiento. Vol. 2 núm.4, Octubre, ISSN: 2588-073X, 2018, pp. 17-31

DOI: 10.26820/recimundo/2.(4).octubre.2018.17-31

URL: http://www.recimundo.com/index.php/es/article/view/337

Editorial Saberes del Conocimiento

Recibido: 15/07/2018

Aceptado: 05/08/2018

Publicado: 30/10/2018

Correspondencia: rubí.1891@ hotmail.com
a. Medico; rubí.1891@ hotmail.com
b. Medico; mianzamat@hotmail.com
c. Medico; kfabiani88@hotmail.com
d. Licenciada en Enfermería; genesisborjatorres@gmail.com 


\section{Eficacia de la escala Alvarado y Ripasa en el diagnóstico oportuno y el control de complicaciones de la apendicitis aguda}

Vol. 2, núm. 4., (2018)

Rubi Alisba Borja Torres; Miguel Andre Zapata Mata; Karla Alejandra Fabiani Portilla; Genesis Ariana Borja Torres

\section{RESUMEN}

La apendicitis aguda es una de las primeras causas de atención quirúrgica en los servicios de urgencia tanto de adultos como de pacientes pediátricos en prácticamente todo el mundo y lógicamente la apendicetomía se establece como el estándar de oro en su tratamiento.

El diagnóstico etiológico del abdomen agudo es con frecuencia difícil y el primer y gran desafío es diferenciar aquellos pacientes con abdomen agudo que requerirán tratamiento médico de aquellos que requerirán una resolución quirúrgica. La causa más común de abdomen agudo quirúrgico que se origina y manifiesta en la fosa iliaca derecha es la apendicitis. Por tal razón, los diferentes exámenes a realizar en un paciente con abdomen agudo de fosa iliaca derecha y de aparente origen inflamatorio estarán dirigidos a confirmar o descartar su origen apendicular. Son múltiples las enfermedades que se manifiestan preferentemente en la fosa iliaca derecha y simulan un cuadro clínico de apendicitis.

El objetivo de esta tesis es determinar la efectividad de utilización de escalas diagnosticas como la de Alvarado y RIPASA para un diagnóstico oportuno de apendicitis aguda y de esa manera prever y controlar las complicaciones de la misma en el Hospital General Milagro Federico Bolaños Moreira durante el periodo 2015-2016 para contribuir con información a las autoridades de la institución y la universidad.

Es necesario aplicar escalas diagnosticas como las descritas en esta tesis para minimizar el tiempo en que se actúa frente a pacientes con patologías como la apendicitis; y de esa manera minimizar presentación de complicaciones.

Palabras claves: Cirugía General, Abdomen agudo, Apendicitis, Escala RIPASA, Escala Alvarado. 


\title{
Eficacia de la escala Alvarado y Ripasa en el diagnóstico oportuno y el control de complicaciones de la apendicitis aguda
}

Vol. 2, núm. 4., (2018)

Rubi Alisba Borja Torres; Miguel Andre Zapata Mata; Karla Alejandra Fabiani Portilla; Genesis

Ariana Borja Torres

\begin{abstract}
Acute appendicitis is one of the leading causes of surgical care in the emergency services of both adults and pediatric patients in virtually the entire world and logically appendectomy is established as the gold standard in its treatment.
\end{abstract}

The etiologic diagnosis of the acute abdomen is often difficult and the first and great challenge is to differentiate those patients with acute abdomen who will require medical treatment of those who will require a surgical resolution. The most common cause of acute surgical abdomen that originates and manifests in the right iliac fossa is appendicitis. For this reason, the different examinations to be performed in a patient with acute abdomen of the right iliac fossa and of an apparent inflammatory origin will be directed to confirm or discard their appendicular origin. Many diseases are manifested preferentially in the right iliac fossa and simulate a clinical picture of appendicitis. The objective of this thesis is to determine the effectiveness of the use of diagnostic scales such as those of Alvarado and RIPASA for a timely diagnosis of acute appendicitis and thus predict and control the complications at the General Milagro Federico Bolaños Moreira Hospital during the period 2015-2016 to contribute information to the authorities of the institution and the university.

It is necessary to apply diagnostic scales as described in this thesis to minimize the time in which patients are treated with pathologies such as appendicitis; And thus minimizing presentation of complications.

Keywords: General Surgery, Acute Abdomen, Appendicitis, RIPASA Scale, Alvarado Scale. 


\section{Eficacia de la escala Alvarado y Ripasa en el diagnóstico oportuno y el control de complicaciones de la apendicitis aguda}

Vol. 2, núm. 4., (2018)

Rubi Alisba Borja Torres; Miguel Andre Zapata Mata; Karla Alejandra Fabiani Portilla; Genesis Ariana Borja Torres

\section{Introducción.}

Abdomen agudo es un síndrome descriptivo muy amplio que define una situación clínica de urgencia, caracterizada por el inicio súbito de síntomas abdominales, consistentes por lo común en dolor, náuseas o vómitos, cambios del ritmo intestinal y/o alteraciones de las funciones genitourinarias. Su gran importancia radica en que es una causa diaria de consulta en las guardias y muchas de las entidades responsables de este síndrome son potencialmente letales si no se tratan de manera precoz. (Reyes-García, Zaldívar-Ramírez, Cruz-Martínez, \& SandovalMartínez, 2012)

La apendicitis aguda es una de las primeras causas de atención quirúrgica en los servicios de urgencia tanto de adultos como de pacientes pediátricos en prácticamente todo el mundo y lógicamente la apendicectomía se establece como el estándar de oro en su tratamiento. (Dubón Peniche, 2014)

El diagnóstico etiológico del abdomen agudo es con frecuencia difícil y el primer y gran desafío es diferenciar aquellos pacientes con abdomen agudo que requerirán tratamiento médico de aquellos que requerirán una resolución quirúrgica. La causa más común de abdomen agudo quirúrgico que se origina y manifiesta en la fosa iliaca derecha es la apendicitis. Por tal razón, los diferentes exámenes a realizar en un paciente con abdomen agudo de fosa iliaca derecha y de aparente origen inflamatorio estarán dirigidos a confirmar o descartar su origen apendicular. Son múltiples las enfermedades que se manifiestan preferentemente en la fosa iliaca derecha y simulan un cuadro clínico de apendicitis. (Bassy Iza, Rodríguez Solís, \& Esteban Dombriz, 2016) 


\section{Eficacia de la escala Alvarado y Ripasa en el diagnóstico oportuno y el control de complicaciones de la apendicitis aguda}

Vol. 2, núm. 4., (2018)

Rubi Alisba Borja Torres; Miguel Andre Zapata Mata; Karla Alejandra Fabiani Portilla; Genesis Ariana Borja Torres

En el diagnóstico de apendicitis, los médicos ponen en la balanza el riesgo de fallar en el diagnóstico, removiendo un apéndice normal en pacientes cuyos signos y síntomas pueden ser atípicos contra el riesgo de perforación del apéndice que podría ocurrir si se opta por una conducta expectante. (López Monclova, Martínez Contreras, \& Zamarrón Ruvalcaba, 2011)

En la mayoría de casos, según Felmer y colaboradores "la decisión quirúrgica se basa en el equilibrio entre el índice de resultados falso-negativo y el índice de perforación a la hora de la exploración”. De tal modo, el realizar un diagnóstico precoz no siempre es fácil debido a la gran variedad de manifestaciones clínicas que están relacionadas con la ubicación de la punta apendicular, edad del paciente y grado de evolución del cuadro. (MONTERO TAPIA \& PINO ANDRAD, 2016)

La perforación apendicular ocurre en aproximadamente el 20 al 30\% de los pacientes con apendicitis. En general, los pacientes con perforación han tenido una mayor duración de los síntomas antes de ser sometidos al tratamiento quirúrgico, lo cual, de nuevo, enfatiza el principio del diagnóstico y tratamiento oportuno. Los pacientes que progresan a la perforación constituyen la mayoría de los casos de morbilidad y mortalidad asociadas con apendicitis aguda. Se ha reportado que los abscesos peri-apendiculares se desarrollan hasta en un 10\% de los pacientes con apendicitis. (Sanabria \& Domínguez, 2013)

Actualmente podríamos considerar la apendicitis como la causa más importante de abdomen agudo quirúrgico teniendo una mayor incidencia en pacientes de sexo masculino entre los 25 a 35 años de edad y el diagnóstico esta dado principalmente por sus hallazgos clínicos, difícil, en especial, en las primeras horas de evolución del cuadro. 


\section{Eficacia de la escala Alvarado y Ripasa en el diagnóstico oportuno y el control de complicaciones de la apendicitis aguda}

Vol. 2, núm. 4., (2018)

Rubi Alisba Borja Torres; Miguel Andre Zapata Mata; Karla Alejandra Fabiani Portilla; Genesis Ariana Borja Torres

Con respecto a los hallazgos en los exámenes de laboratorio, se puede decir que ninguna prueba confirma o excluye el diagnostico cuando se utilizan de manera aislada, ya sean alteraciones de la serie blanca (leucocitosis en el $80 \%$ o leucopenia en el $20 \%$ de los casos), PCR, o marcadores nuevos como Lactoferrina, Calprotectina, etc.

Esto ha dado lugar a numerosas investigaciones para identificar hallazgos clínicos, de laboratorio y por supuesto imagenológicos con mayor certeza diagnostica y así mismo el desarrollo de sistemas de puntuación clínica como guía para el personal médico con el fin de hacer un diagnóstico correcto y de esa manera reducir el retraso en una adecuada y oportuna evaluación del paciente, disminuyendo las tasas de apendicectomías negativas.

Todos estos datos apoyan la importancia de la apendicitis aguda como problema de salud en la sociedad, y se debe considerar a los centros médicos de atención primaria de salud como protagonistas activos del diagnóstico precoz y así mismo se debe considerar la responsabilidad del médico en sospechar o diagnosticar y no dejar pasar por alto esta patología y por ende dar lugar a complicaciones que aumenten la mortalidad en los pacientes.

La escala Alvarado es probablemente la de mayor difusión y aceptación en los servicios de urgencias a nivel mundial, con una sensibilidad del 68\% y especificidad del 87\%, (Quesada \& Ival, 2015) sin embargo muchas veces es sobreestimada y poco utilizada en las salas de urgencias de nuestro país, lo que pone en riesgo la oportuna evaluación de los pacientes con síndrome doloroso abdominal.

Esta escala clasifica en 3 grupos con la probabilidad de tener apendicitis: 


\section{Eficacia de la escala Alvarado y Ripasa en el diagnóstico oportuno y el control}

de complicaciones de la apendicitis aguda

Vol. 2, núm. 4., (2018)

Rubi Alisba Borja Torres; Miguel Andre Zapata Mata; Karla Alejandra Fabiani Portilla; Genesis

Ariana Borja Torres

- Riesgo bajo: probabilidad de apendicitis del 7\%.

- Riesgo intermedio: probabilidad de apendicitis del 57\%.

- Riesgo alto: probabilidad de apendicitis del $90 \%$.

Recientemente se está aplicando una escala mejorada para el diagnóstico precoz de la apendicitis aguda, que es la escala RIPASA con una mejor sensibilidad de $98 \%$ y especificidad de $83 \%$, y de acuerdo al puntaje se considera el manejo del paciente.

- $\quad<5$ puntos: improbable.

- $5-7$ puntos: baja probabilidad.

- 7 - 11 puntos: alta probabilidad.

- > 12 puntos: diagnóstico de apendicitis.

Al aplicar cualquiera de las dos escalas en las áreas de urgencia de nuestros hospitales incrementaríamos la certeza diagnóstica de esta patología, con el consecuente beneficio de los pacientes y la reducción de su morbimortalidad.

El objetivo de la presente investigación es determinar la eficacia de las escalas Alvarado y RIPASA en el diagnóstico oportuno de la Apendicitis Aguda al momento de ingreso de los pacientes al área o servicio de emergencia.

\section{Metodología.}

En esta investigación se evalúan los casos de pacientes operados por apendicitis aguda por método indirecto sin utilización de escalas diagnósticas, con informe histopatológico para 


\section{Eficacia de la escala Alvarado y Ripasa en el diagnóstico oportuno y el control de complicaciones de la apendicitis aguda}

Vol. 2, núm. 4., (2018)

Rubi Alisba Borja Torres; Miguel Andre Zapata Mata; Karla Alejandra Fabiani Portilla; Genesis Ariana Borja Torres

esa enfermedad, mediante un estudio descriptivo retrospectivo, en el cual se recolectó la información de las historias clínicas. Y método directo los casos de aquellos pacientes que ingresan a emergencia y se les aplica diagnostico basado en cada una de las escalas por medio de hoja de recolección de datos.

\section{Descripción de procedimientos}

El estudio se realizó a través de la observación de pacientes mayores de 15 años que acudieron a emergencia del Hospital General Milagro "FEDERICO BOLAÑOS MOREIRA" con un dolor abdominal sugestivo de apendicitis, de acuerdo a los objetivos planteados e investigación se obtendrá la información basándose en la investigación de reportes del Área de Cirugía General que se encuentran en el servicio de estadísticas, para recolectar los números de historia clínica de los pacientes con diagnóstico de abdomen agudo que hayan sido intervenido quirúrgicamente por apendicitis aguda y de esta forma revisar cada una de las historias clínicas y obtener información necesaria, a los mismos se le realiza el examen físico directo, exámenes de laboratorio e imagen; estos pacientes al examen físico y elaboración de la historia clínica dieron positivo a la mayor parte de signos y síntomas para apendicitis, entre ellos dolor a la presión en FID, dolor al rebote, anorexia, náusea, vómito , además se evidenció en exámenes de laboratorio la elevación de los leucocitos por encima de 12000 x mm3 y EMO negativo.

Estos datos fueron registrados en el formulario de recolección de datos.

Los pacientes que acudieron por el servicio de emergencia y tras ser evaluados fueron incluidos, los que tras la valoración clínica y los exámenes de laboratorio e imagen se sospechó 


\section{Eficacia de la escala Alvarado y Ripasa en el diagnóstico oportuno y el control}

de complicaciones de la apendicitis aguda

Vol. 2, núm. 4., (2018)

Rubi Alisba Borja Torres; Miguel Andre Zapata Mata; Karla Alejandra Fabiani Portilla; Genesis

Ariana Borja Torres

apendicitis aguda; estos pacientes fueron intervenidos quirúrgicamente y la muestra patológica fue enviada a anatomía patológica para su valoración; los datos fueron recogidos en el periodo pre quirúrgico mediante el uso de un formulario de recolección de la información para las variables demográficas, durante el periodo post quirúrgico se recolectaron variables como el diagnóstico macroscópico de apendicitis y se revisó el informe de anatomía patológica.

Especial revisión en formulario de emergencia 008, junto a la hoja de ingreso, donde encontramos datos para identificar complicaciones y establecer diagnósticos. En el presente estudio se utilizara tabulación de datos por medio de Microsoft Excel.

\section{Resultados.}

Tras la recolección de los datos y la validación de la información se creó una base de datos; el análisis se lo llevó a cabo mediante técnicas descriptivas como son tablas de frecuencia simples, donde según el tipo de variable se obtuvo estadísticos como media; también se analizan los resultados mediante el cruce de variables para determinar asociación.

Los datos fueron recolectados de 300 pacientes durante el periodo de estudio, en los que se utilizó escala Alvarado, y escala RIPASA; se contó también con la disponibilidad de los exámenes anatomopatológicos de manera adecuada, los resultados de esta investigación se presentan a continuación:

\section{Distribución de 800 pacientes con Dx. de Abdomen Agudo por patología}




\section{Eficacia de la escala Alvarado y Ripasa en el diagnóstico oportuno y el control de complicaciones de la apendicitis aguda}

Vol. 2, núm. 4., (2018)

Rubi Alisba Borja Torres; Miguel Andre Zapata Mata; Karla Alejandra Fabiani Portilla; Genesis Ariana Borja Torres

- ANALSIS: Se tomaron 800 casos de Abdomen Agudo que fueron ingresados por emergencia al Hospital IESS Milagro, de los cuales 300 casos que fueron escogidos para la muestra de este estudio resultaron ser Apendicitis Aguda representando el 37.5\%; la segunda patología de presentación fue la Infección de vías urinarias con el $26 \%$ de los casos y posteriormente con diferencia del $0.5 \%$ la enfermedad pélvica inflamatoria y la colecistitis con $13,5 \%$ y $14 \%$ respectivamente. Hay que denotar la presencia también de Embarazos ectópicos, se presentaron 12 casos que representa el $1.5 \%$ de los 800 admitidos por Dx. de Abdomen agudo.

\section{Distribución de 300 pacientes apendicectomizados según sus edades y sexo}

- ANALSIS: Se puede observar que un proceso patológico apendicular de tipo quirúrgico se presentó mayoritariamente en pacientes masculinos con alrededor de 172 pacientes de todo el estudio realizado lo que equivale al 57\%. Así también se observa una mayor incidencia de apendicitis aguda entre la 2da y 3era década de la vida de los cuales igualmente la mayoría era de pacientes masculinos.

\section{Distribución de 300 pacientes apendicectomizados según su diagnóstico histológico.}

- ANALSIS: El resultado anatomopatológicos reveló que el 44,3\% de los pacientes presentaron un apéndice en fase inflamatoria al momento de la cirugía y que solo el 2,3\% de piezas apendiculares extirpadas se encontraban sin aparente estado patológico.

Distribución de 300 pacientes apendicectomizados según características del cuadro clínico. 
Eficacia de la escala Alvarado y Ripasa en el diagnóstico oportuno y el control de complicaciones de la apendicitis aguda

Vol. 2, núm. 4., (2018)

Rubi Alisba Borja Torres; Miguel Andre Zapata Mata; Karla Alejandra Fabiani Portilla; Genesis Ariana Borja Torres

- ANALISIS: Analizando los componentes clínicos de los 300 pacientes, se pudo observar que el síntoma más frecuente es la anorexia presentándose en el 90\%, seguido de la migración del dolor con el 88,3\% y posteriormente de uno de sus signos patognomónicos como es el signo de Mc Burney con el 87,7\%. Aunque la sintomatología parece abundante hay que recordar que las presentaciones atípicas se pueden presentar.

Distribución de 300 pacientes apendicectomizados según valores de laboratorio encontrados.

- ANALSIS: Se encontró leucocitosis en el 90,3\% de los casos, con desviación a la izquierda de la serie de neutrófilos en el $86 \%$ de los pacientes y el EMO fue infeccioso solo en el $17 \%$ de los casos.

\section{Parámetros de laboratorio presentados en el total de pacientes}

- ANALISIS: Según la escala de Alvarado, el 65,3\% de los pacientes presentaron un puntaje que daba como resultado apendicitis aguda (9-10 puntos); el 22,3\% como probable apendicitis (7-8 puntos); en lo que respecta a los puntajes que indicaban negativo se encontró una frecuencia del 4,3\%.

Distribución de 300 pacientes apendicectomizados según valoración según Score de RIPASA.

- ANALSIS: Tras valorar los puntajes obtenidos para el Score de RIPASA encontramos que el 68,3\% de la población presentó un puntaje diagnóstico de apendicitis; el 29,7\% 


\section{Eficacia de la escala Alvarado y Ripasa en el diagnóstico oportuno y el control de complicaciones de la apendicitis aguda}

Vol. 2, núm. 4., (2018)

Rubi Alisba Borja Torres; Miguel Andre Zapata Mata; Karla Alejandra Fabiani Portilla; Genesis Ariana Borja Torres

alta probabilidad y el $1,7 \%$ baja probabilidad; para los fines de nuestro trabajo de investigación encontramos que el $0,3 \%$ ( 1 caso) presentó puntajes con niveles que indicaron improbable.

Distribución de 300 pacientes apendicectomizados según presentación de complicaciones.

- ANALISIS: Posterior al procedimiento quirúrgico, durante la hospitalización de los pacientes, la complicación más frecuente presentada es la infección de herida quirúrgica con el 28,7\% de los casos, seguido del absceso de pared. En este estudio ninguno de los pacientes presento dehiscencia del muñón apendicular o hemorragias y solo 2 casos presentaron fistulización colactánea lo que corresponde al $0.6 \%$ de los casos; en general más de la mitad de los casos no presentaron complicación alguna.

\section{ANÁLISIS INFERENCIAL}

Distribución de 300 pacientes apendicectomizados en presentación de complicaciones según la edad y sexo.

- ANALISIS: Analizando la tabla podemos determinar que de la muestra de 300 pacientes, la edad más frecuente de complicaciones así como de presentación de apendicitis aguda es entre los 20-34 años de edad con 70 pacientes con herida quirúrgica infectada sin mucha diferencia entre sexo masculino y femenino. Y la presentación de fistula colo- 
Eficacia de la escala Alvarado y Ripasa en el diagnóstico oportuno y el control de complicaciones de la apendicitis aguda

Vol. 2, núm. 4., (2018)

Rubi Alisba Borja Torres; Miguel Andre Zapata Mata; Karla Alejandra Fabiani Portilla; Genesis Ariana Borja Torres

cutanea cuya incidencia fue del $0.4 \%$ tanto en hombres como en mujeres de más de 35 años de edad. Hay que recalcar que la fistulización de estos pacientes fue el resultado de demora en el diagnóstico oportuno de estos pacientes.

Distribución de 300 pacientes apendicectomizados en presentación de complicaciones según el laboratorio.

- ANALISIS: Al analizarla podemos observar que tanto si existe complicaciones o no en nuestra muestra de 300 pacientes se presentó leucocitosis con predominio de neutrófilos en cerca del 90\% de los casos y el EMO fue infeccioso en el 17\% de los casos.

Distribución de 300 pacientes apendicectomizados en presentación de complicaciones según las escalas diagnósticas.

- ANALISIS: Podemos determinar que aquellos pacientes diagnosticados con escala RIPASA cuyos resultados dieron positivo para apendicitis, cerca del $68 \%$ no presentaron complicación alguna y el 22\% tuvo infección de herida quirúrgica. Así mismo podemos decir que no se presentaron complicaciones en aquellos pacientes cuyo score RIPASA dio baja probabilidad de Apendicitis.

Distribución de 300 pacientes apendicectomizados en presentación de complicaciones según las escala Alvarado. 


\section{Eficacia de la escala Alvarado y Ripasa en el diagnóstico oportuno y el control de complicaciones de la apendicitis aguda}

Vol. 2, núm. 4., (2018)

Rubi Alisba Borja Torres; Miguel Andre Zapata Mata; Karla Alejandra Fabiani Portilla; Genesis Ariana Borja Torres

- ANALISIS: Podemos observar en esta tabla que aquellos pacientes diagnosticados con escala Alvarado y cuyos resultados dieron positivo para apendicitis, cerca del $38 \%$ no presentaron complicación alguna y el $16 \%$ si la tuvo como es el caso de herida quirúrgica infectada. Así mismo podemos decir que no se presentaron complicaciones en aquellos pacientes cuyo score Alvarado dio negativo para Apendicitis aguda.

\section{Conclusiones.}

- Se analizaron 300 casos de apendicectomías, de las cuales el 52\% se presentaron en pacientes de entre 20-34 años y de los cuales se presenta el 57\% de los casos en el sexo masculino.

- El síntoma más frecuentemente encontrado en pacientes con apendicetomía es la anorexia en el 90\% de los casos, seguido de la migración del dolor.

- Según la escala de Alvarado, el 65,3\% de los pacientes presentaron un puntaje que daba como resultado apendicitis aguda (9-10 puntos); mientras que con la escala RIPASA, el 68,3\% de los pacientes presentaron un diagnóstico certero de apendicitis aguda.

- El 4,3\% de los pacientes con sospecha de apendicitis con la escala de Alvarado y el 0,3\% con el Score de RIPASA resultaron ser diagnósticos improbables de apendicitis según sus respectivas escalas. Si bien es cierto se observa que la eficacia de la escala RIPASA es mayor con respecto a un diagnóstico confiable de apendicitis; la rapidez con la que se 


\section{Eficacia de la escala Alvarado y Ripasa en el diagnóstico oportuno y el control}

de complicaciones de la apendicitis aguda

Vol. 2, núm. 4., (2018)

Rubi Alisba Borja Torres; Miguel Andre Zapata Mata; Karla Alejandra Fabiani Portilla; Genesis

Ariana Borja Torres

puede evaluar según la escala Alvarado la hace la más usada por nuestros médicos en área de emergencias.

- La prevalencia de apendicectomías no enfermas fue del 2,3\% que equivalen a 7 de 300 casos estudiados; si bien es cierto que este estudio no trata acerca de apendicectomías en blanco, es importante tomar en cuenta que se siguen presentando casos de apendicectomías no enfermas confirmadas por anatomopatología y ya que la patología apendicular es una de las más frecuentes en nuestras emergencias; es un tema que no se debería dejar de lado y por ende incentivar de una u otra forma para profundizar de mejor manera en este tema.

- El 58\% de los pacientes estudiados no presentaron complicación alguna durante el acto quirúrgico o durante el post-operatorio; pero se pudo identificar que de los pacientes que si presentaron complicaciones la de más alta frecuencia es la herida infectada con el 28,7\% de los casos. Se debería determinar si la causa de esto es por un diagnóstico tardío de patología apendicular o por un mal manejo de las mismas por parte de los cirujanos.

\section{Bibliografía.}

Bassy Iza, N., Rodríguez Solís, J., \& Esteban Dombriz, M. (2016). ABDOMEN AGUDO. En TRATADO de GERIATRÍA para residentes. Madrid: Sociedad Española de Geriatría y Gerontología.

Dubón Peniche, M. (2014). Apendicitis aguda, su diagnóstico y tratamiento. Revista de la Facultad de Medicina (México), 57(4), 51-57.

López Monclova, J., Martínez Contreras, A., \& Zamarrón Ruvalcaba, A. (2011). Apendicectomías negativas y su relación con los estudios de imagen. Cirujano general, 33(4), 222-226. 


\section{Eficacia de la escala Alvarado y Ripasa en el diagnóstico oportuno y el control de complicaciones de la apendicitis aguda}

Vol. 2, núm. 4., (2018)

Rubi Alisba Borja Torres; Miguel Andre Zapata Mata; Karla Alejandra Fabiani Portilla; Genesis Ariana Borja Torres

MONTERO TAPIA, E., \& PINO ANDRAD, R. (2016). APENDICECTOMÍAS NO ENFERMAS EN EL HOSPITAL VICENTE CORRAL MOSCOSO Y HOSPITAL JOSÉ CARRASCO ARTEAGA, CUENCA 2014. Cuenca: UNIVERSIDAD DE CUENCA.

Quesada, S., \& Ival, P. (2015). La escala de Alvarado como recurso clínico para el diagnóstico de la apendicitis aguda. Revista Cubana de Cirugía, 54(2), 121-128.

Reyes-García, N., Zaldívar-Ramírez, F., Cruz-Martínez, R., \& Sandoval-Martínez, M. (2012). Precisión diagnóstica de la escala RIPASA para el diagnóstico de apendicitis aguda: análisis comparativo con la escala de Alvarado modifi cada. Cirujano General, 34(2), 101-106.

Sanabria, Á., \& Domínguez, L. (2013). Tiempo de evolución de la apendicitis y riesgo de perforación. Rev Colomb Cir, 28(1), 24-30. 